\title{
Impairment of Sulfite Reductase Decreases Oxidative Stress Tolerance in Arabidopsis thaliana
}

\author{
Meiping Wang, Yunli Jia, Ziwei Xu and Zongliang Xia* \\ College of Life Science, Henan Agricultural University, Zhengzhou, China
}

As an essential enzyme in the sulfate assimilation reductive pathway, sulfite reductase $(\mathrm{SiR})$ plays important roles in diverse metabolic processes such as sulfur homeostasis and cysteine metabolism. However, whether plant SiR is involved in oxidative stress response is largely unknown. Here, we show that SiR functions in methyl viologen (MV)-induced oxidative stress in Arabidopsis. The transcript levels of SiR were higher in leaves, immature siliques, and roots and were markedly and rapidly up-regulated by MV exposure. The SiR knock-down transgenic lines had about 60\% residual transcripts and were more susceptible than wild-type when exposed to oxidative stress. The severe damage phenotypes of the SiR-impaired lines were accompanied by increases of hydrogen peroxide $\left(\mathrm{H}_{2} \mathrm{O}_{2}\right)$, malondialdehyde (MDA), and sulfite accumulations, but less

OPEN ACCESS

Edited by:

Manuel González-Guerrero, Technical University of Madrid, Spain

Reviewed by:

Fabio Francesco Nocito, University of Milan, Italy

Manuel Tejada-Jimenez,

University of Córdoba, Spain

*Correspondence:

Zongliang Xia xiazI08@163.com

Specialty section: This article was submitted to Plant Nutrition,

a section of the journal

Frontiers in Plant Science

Received: 04 October 2016 Accepted: 22 November 2016 Published: 02 December 2016

Citation:

Wang M, Jia Y, Xu Z and Xia Z (2016) Impairment of Sulfite Reductase Decreases Oxidative Stress Tolerance in Arabidopsis thaliana. Front. Plant Sci. 7:1843. doi: 10.3389/fpls.2016.01843 amounts of glutathione (GSH). Interestingly, application of exogenous GSH effectively rescued corresponding MV hypersensitivity in SiR-impaired plants. GRT-PCR analysis revealed that there was significantly increased expression of several sulfite metabolismrelated genes in SiR-impaired lines. Noticeably, enhanced transcripts of the three APR genes were quite evident in SiR-impaired plants; suggesting that the increased sulfite in the SiR-impaired plants could be a result of the reduced SiR coupled to enhanced APR expression during oxidative stress. Together, our results indicate that $S i R$ is involved in oxidative stress tolerance possibly by maintaining sulfite homeostasis, regulating GSH levels, and modulating sulfite metabolism-related gene expression in Arabidopsis. SiR could be exploited for engineering environmental stress-tolerant plants in molecular breeding of crops.

\section{Keywords: sulfite reductase, methyl viologen, oxidative stress, glutathione}

\section{INTRODUCTION}

The assimilatory reduction of inorganic sulfate into organic sulfur compounds proceeds via a highly coordinated mechanism in higher plants (Khan et al., 2010). First, sulfate is adenylated by ATP sulfurylase to adenosine 5-phosphosulfate (APS). Next, APS is reduced to sulfite by the 5-phosphosulfate reductase (APR). The toxic intermediate sulfite is further reduced by sulfite reductase $(\mathrm{SiR})$ to sulfide, which is then incorporated into Cys and other sulfur-containing amino acids and sulfolipids (Leustek and Saito, 1999; Leustek et al., 2000; Nakayama et al., 2000). As an essential enzyme in the sulfate reduction pathway, SiR plays important roles in diverse metabolic processes such as sulfur detoxification and cysteine metabolism (Wirtz et al., 2004; Brychkova et al., 2007; Lang et al., 2007). 
Plant SiR is a soluble protein containing one $(4 \mathrm{Fe}-4 \mathrm{~S})$ cluster and one siroheme that catalyzes the six-electron reduction of sulfite to sulfide (Lewandowska and Sirko, 2008). SiR exists as a single copy in the genome of Arabidopsis and is localized exclusively in the plastids (Sekine et al., 2007). SiR acts as a sulfur assimilation enzyme and a chloroplast nucleoid binding protein, indicating that it is essential for the assimilatory sulfate reduction and growth and development of plants (Sekine et al., 2007; Kang et al., 2010; Khan et al., 2010). It has recently demonstrated that SiR plays a role in protecting Arabidopsis or tomato plants against sulfite toxicity (Yarmolinsky et al., 2013). Further investigation showed that knockdown expression of SiR resulted in accelerated leaf senescence in tomato plants (Yarmolinsky et al., 2014).

Environmental stresses including abiotic and biotic stress provoke cellular redox imbalances and generate excessive reactive oxygen species (ROS) such as hydroxyl radicals and superoxide ions, which result in oxidative stress in plants (Gechev et al., 2006). When plant cells cannot remove excess ROS promptly, leaves become pale and necrotic. To maintain redox balance and to protect against oxidative stress, plants have evolved a ROS-scavenging system to eliminate excess ROS, including non-enzymatic antioxidants, such as ascorbic acid, glutathione (GSH) and carotenoids, and ROS-removing enzymes. It has been recently shown that impaired-SiR tomato plants significantly decreased GSH levels and led to early leaf senescence (Yarmolinsky et al., 2014). As we know, GSH is both an important reduced sulfur sink and a regulator of sulfur assimilation (Hell, 1997). Also, it plays an important role in protecting plants against oxidative stress (Alscher, 1989; Noctor et al., 1998). However, whether plant SiR participates in oxidative stress response is unclear. In this study, we provide genetic evidence that $S i R$ functions in methyl viologen (MV)-induced oxidative stress in Arabidopsis.

\section{MATERIALS AND METHODS}

\section{Plant Materials and Growth Conditions}

Arabidopsis thaliana ecotype Columbia (Col-0) was used as the wild type in this study. The wild type and RNAi transgenic seeds were surface sterilized and germinated on plates containing $1 / 2$ Murashige and Skoog (MS) medium. Seeds were stratified at $4^{\circ} \mathrm{C}$ in darkness for 3 days and then transferred to a growth chamber at $22^{\circ} \mathrm{C}$ with a 16 -h-light/8-h-dark photoperiod. After 1 week, the seedlings were transferred to sterilized low-nutrient soil to obtain fully grown plants. Plants were grown in a growth room at approximately $22^{\circ} \mathrm{C}, 70-80 \%$ relative humidity, a photoperiod of $16 \mathrm{~h} / 8 \mathrm{~h}$ (day/night) and light intensity of $200 \mu \mathrm{mol} \mathrm{m}^{-2} \mathrm{~s}^{-1}$, as described before (Xia et al., 2016).

\section{Real-Time PCR Analysis}

Real-time PCR was used to determine expression pattern of $S i R$ in different organs and transcript levels of several sulfite network genes ( $S i R, S O, S Q S 1, A P R 1, A P R 2$, and $A P R 3)$ in response to $\mathrm{MV}$ treatment. Total RNA extraction, first-strand cDNA synthesis and qRT-PCR with gene-specific primers (Supplementary Table S1) were conducted as described previously (Xia et al., 2016). The Arabidopsis Actin2 transcript was used as an internal control to quantify the relative transcript levels as described (Livak and Schmittgen, 2001; Xia et al., 2016). We had previously compared Arabidopsis EF-1a, Actin2, and Tubulin as internal controls and found that Actin2 is more stable than the others as a reference gene in our pilot experiment. All qRT-PCR experiments were performed with three biological and three technical replicates.

\section{Construction of Plant Expression Vectors and Development of RNAi Transgenic Arabidopsis Lines}

For the RNA interference (RNAi) construct, a 369-bp-length fragment of SiR cDNA was amplified using primers SiR-F and SiR-R (Supplementary Table S1) and introduced as sense and antisense into the binary vector pFGM with BamHI and XbaI, or NcoI and SwaI restriction sites, resulting in the transformation construct pFGM-35S:SiR-RNAi.

The binary construct was introduced into Agrobacterium tumefaciens (strain GV3101) and then transformed into Arabidopsis (Col-0) via the floral dip method (Clough and Bent, 1998). Transformed lines were selected by antibiotic resistance and verified by PCR analysis. Homozygous Arabidopsis SiRmodified lines that contained single-site transgene insertions were identified and maintained growth to set seeds until $\mathrm{T}_{3}$ generation. Homozygous $\mathrm{T} 3$ lines were used for further experiments.

\section{Analysis of SiR RNAi Transgenic Arabidopsis Lines for Oxidative Stress Tolerance}

The WT and transgenic lines (Ri-1, Ri-4 and Ri-6) were cultured in $1 / 2 \mathrm{MS}$ medium under a $16 \mathrm{~h}$ light $/ 8 \mathrm{~h}$ dark cycle at $22^{\circ} \mathrm{C}$ for 1 week, and then the plants were transplanted into small pots with soil (four plants per pot, and two pots for each line) and grown for 4 weeks under standard growth conditions. Fiveweek-old WT and RNAi lines were sprayed with $20 \mu \mathrm{M}$ of MV. Three replicates each consisting of two pots of seedlings from each line were included for both $\mathrm{MV}$ treatment and $\mathrm{H}_{2} \mathrm{O}$ treated controls. The whole experiment was repeated three times. The remaining chlorophyll content of WT and RNAi lines was determined 3 days after treatment. Leaf damage level (LDL) was determined as the ratio of damaged area divided by the whole leaf area. The relative damage level (\%) was calculated as the mean of LDL of five leaves from each plant.

\section{Determination of MDA and $\mathrm{H}_{2} \mathrm{O}_{2}$ Contents}

MDA content was determined as described previously (Draper and Hadley, 1990; Huo et al., 2016). $\mathrm{H}_{2} \mathrm{O}_{2}$ content was assayed according to our previously used method (Xia et al., 2012). The absorbance of the resulting solution was measured at $415 \mathrm{~nm}$ and the $\mathrm{H}_{2} \mathrm{O}_{2}$ concentration was determined using a standard curve plotted with standard concentrations of $\mathrm{H}_{2} \mathrm{O}_{2}$. In both experiments, three independent biological replicates were conducted, and three times were done in each independent assay. 


\section{Determination of SiR Activity, Sulfite, and Glutathione Contents}

Leaf samples were extracted and protein concentration was determined as described previously (Bradford, 1976; Xia et al., 2012). The assay solution contained $10 \mathrm{mM}$ DTT, $25 \mathrm{mM}$ HEPES, $\mathrm{pH}$ 7.8, $5 \mathrm{mM}$ OAS, 5 units of OAS-TL, $15 \mathrm{mM} \mathrm{Na} \mathrm{S}_{2} \mathrm{O}_{4}$, $30 \mathrm{mM} \mathrm{NaHCO}, 1 \mathrm{mM} \mathrm{Na} \mathrm{SO}_{3}$, and $5 \mathrm{mM} \mathrm{MV}$ along with the crude leaf extract. SiR activity was determined according to the assay of Khan et al. (2010). Sulfite levels of leaf samples were determined using ion-exchange chromatograph system as described (Brychkova et al., 2012; Xia et al., 2012). GSH was determined according to the method as described by Griffith (1980). For each experiment, three replicates were conducted for each test sample and the experiment was repeated three times.

\section{Accession Numbers}

Sequence data from this article can be found in the GenBank/EMBL data libraries under the following accession numbers: SiR, At5g04590; SO, At3g01910; SQS1, At4g33030; APR1, At4g04610; APR2, At1g62180; APR3, At4g21990.

\section{RESULTS}

\section{Transcript Profiles of SiR in Arabidopsis Organs and During Oxidative Stress}

The transcriptional pattern of SiR was examined in five organs of Arabidopsis (roots, stems, leaves, flowers, and immature siliques). The $S i R$ transcript levels were significantly high in leaves and immature siliques (Figure 1A). In contrast, $S i R$ transcripts were low in stems. The relative expression in leaves was three times greater than that in the stems (Figure 1A).

Time-course analysis of $\mathrm{SiR}$ transcript levels in Arabidopsis plants in response to oxidative stress was performed by qRT-PCR (Figure 1B). The transcript levels of SiR were increased rapidly after $1 \mathrm{~h}$, and reached a maximal level at $12 \mathrm{~h}$ (about sixfold increase in transcripts) during $48 \mathrm{~h}$ period of $\mathrm{MV}$ treatment (Figure 1B). In contrast, no significant difference was observed under control conditions (Figure 1B). These data suggest that $\mathrm{SiR}$ could be involved in oxidative stress response.

\section{Generation of SiR Knockdown Arabidopsis Plants}

To further investigate the role of $S i R$ in oxidative stress tolerance, transgenic Arabidopsis plants under-expressing SiR were generated (Figure 2A). Due to early seedling lethal caused by SiR inhibition (Khan et al., 2010), only five independent transgenic lines (T1; Ri-1, Ri-3, Ri-4, Ri-6 and Ri-11) were identified by antibiotic-resistance analysis and PCR using specific primers (Supplementary Table S1). qRT-PCR analysis of the T1 generation revealed that these five RNAi lines exhibited 30-70\% reductions in $\mathrm{SiR}$ transcripts when compared with wild type (Figure 2A). Accordingly, these RNAi lines showed significantly reduced levels of $\mathrm{SiR}$ activity (30-68\% of the wild type level; Figure 2B). However, the RNAi line Ri-11 showed severe growth retardation and could not be used for screening homozygous line

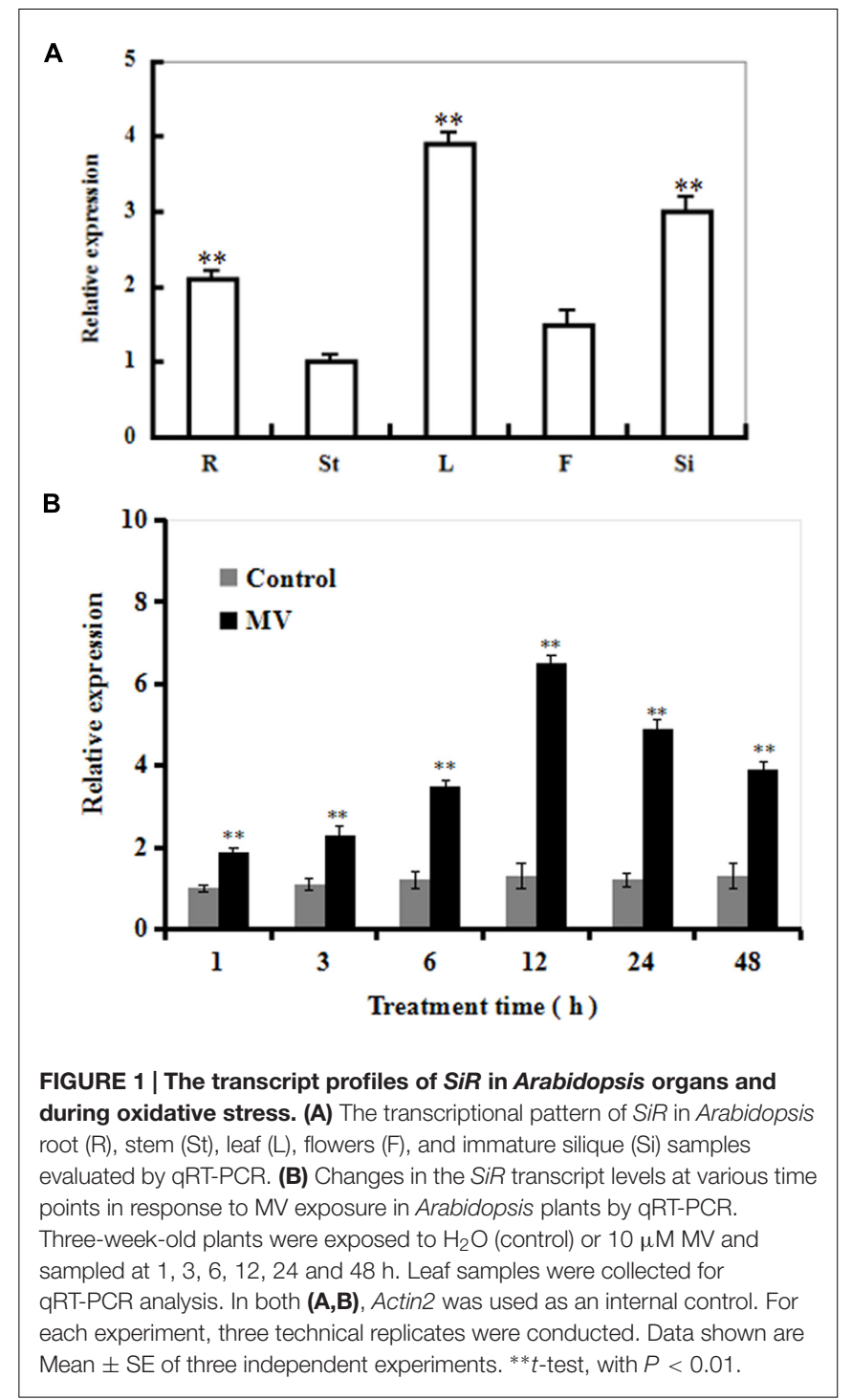

because of strong suppression in SiR expression (Figures 2A,B). Among the remaining four lines, three homozygous lines (Ri-1, $\mathrm{Ri}-4$ and $\mathrm{Ri}-6)$ had $55-70 \%$ of the wild type levels, which were chosen for further experiments.

\section{Response of SiR Under-Expressing Arabidopsis Plants to Oxidative Stress}

To characterize the performance of $S i R$ under-expressing Arabidopsis under MV-induced oxidative stress, T3 seeds of the three homozygous RNAi lines were directly sown in soil. $20 \mu \mathrm{M}$ of MV was applied to 5-week-old seedlings by spraying directly onto the leaves. Three days after MV spraying, the RNAi transgenic plants showed relative higher necrosis and wilting than the wild-type plants (Figure 3A). Accordingly, the mean LDL of the RNAi lines was about twofold higher than that in the WT (Figure 3B) and the remaining chlorophyll content in the RNAi lines was significantly lower than that in WT plants ( $\sim 36 \%$ on the average; Figure $3 \mathrm{C}$ ). These results demonstrate 

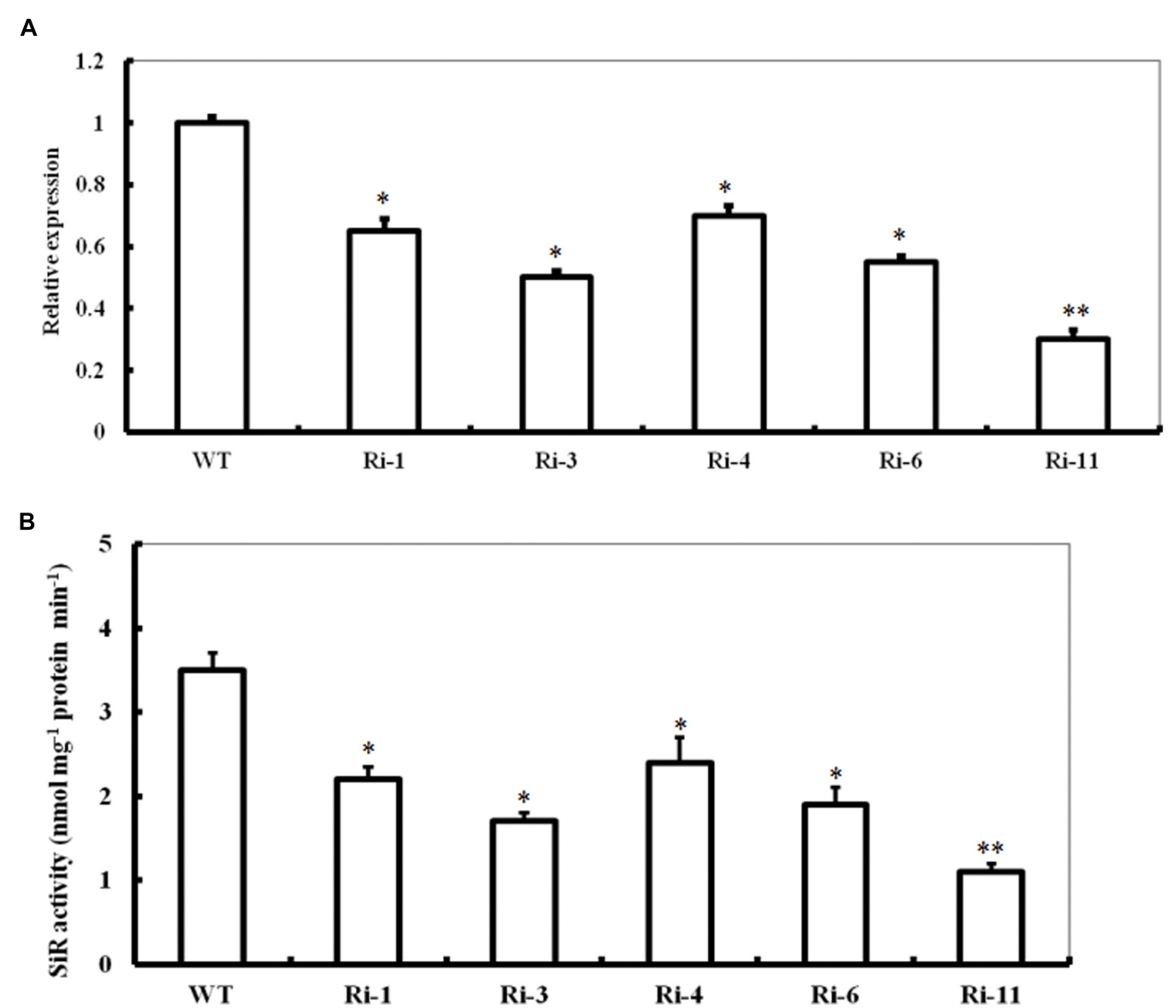

FIGURE 2 | Expression levels and activity of SiR in SiR-modified Arabidopsis plants. (A) Transcript levels of SiR in the WT and five RNA interference lines (named Ri-1, Ri-3, Ri-4, Ri-6, and Ri-11) determined by qRT-PCR. (B) Total SiR activity in leaf extracts from WT and RNAi lines. In both histograms above, values are mean \pm SE. The asterisks indicate significance of the difference from the corresponding control values determined by Student's $t$-test $(* * t$-test, with $P<0.01$; *t-test, with $P<0.05)$.

that impairment of $\mathrm{SiR}$ in Arabidopsis plants decreases tolerance to MV-induced oxidative stress.

\section{Under-Expression of SiR Increases MDA and $\mathrm{H}_{2} \mathrm{O}_{2}$ Accumulations under Oxidative Stress}

Reduced oxidative tolerance in SiR RNAi lines prompted us to detect the differences in lipid peroxidation. Malondialdehyde (MDA), a product of lipid peroxidation was measured between the WT and RNAi plants 3 days after MV treatment. The MDA levels in the RNAi lines (149, 133, and 190\% increases for RNAi$1,-4$, and -6 , respectively) were significantly higher than in the WT (only $80 \%$ increase), suggesting that the RNAi plants suffered more membrane damage than the wild type (Figure 4A). By contrast, in the presence of GSH, the MDA levels in the RNAi lines were only $70 \%$ increase on average after $\mathrm{MV}$ exposure (Figure 4A).

The SiR knockdown plants had higher MDA levels under oxidative stress, implying that they may be subjected to more serious oxidative damage than the WT. Therefore, it was of interest to detect ROS accumulation in the WT and RNAi lines during oxidative stress. Quantitative determination of $\mathrm{H}_{2} \mathrm{O}_{2}$ accumulation was performed in 3-day MV-treated leaves along with controls from RNAi and WT plants. As shown in Figure $4 \mathrm{~B}, \mathrm{H}_{2} \mathrm{O}_{2}$ content increased in both WT and transgenic lines after oxidative stress. However, RNAi lines accumulated higher levels of $\mathrm{H}_{2} \mathrm{O}_{2}$ (200\% increase averagely) relative to WT (130\% increase) after MV treatment (Figure 4B). By contrast, in the presence of GSH, there was no significant difference in $\mathrm{H}_{2} \mathrm{O}_{2}$ content between RNAi and WT plants after MV exposure (Figure 4B). These physiological indices demonstrated that higher ROS accumulation and lipid peroxidation in the RNAi transgenic lines may be correlated to their decreased tolerance to oxidative stress and was attenuated by GSH.

\section{Sulfite and Glutathione (GSH) Accumulations in SiR Under-Expressing Lines upon MV Exposure}

To monitor in planta changes in the levels of substrate and products upon MV exposure, sulfite and S-metabolite GSH 
A
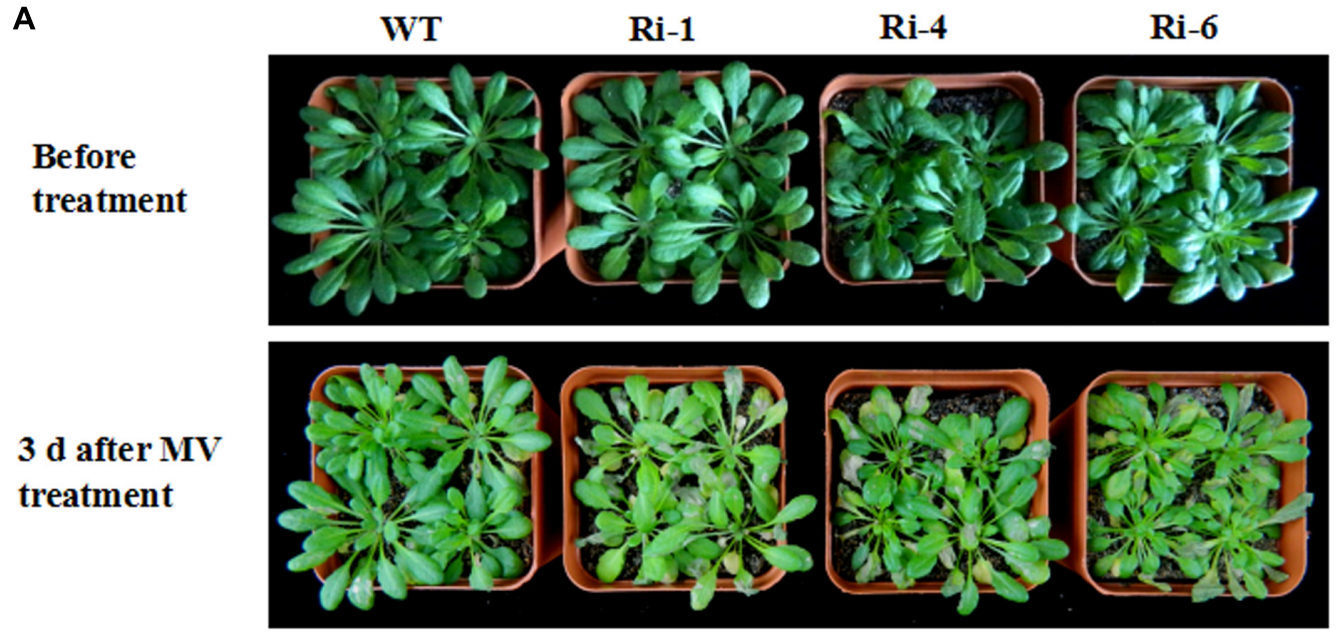

B

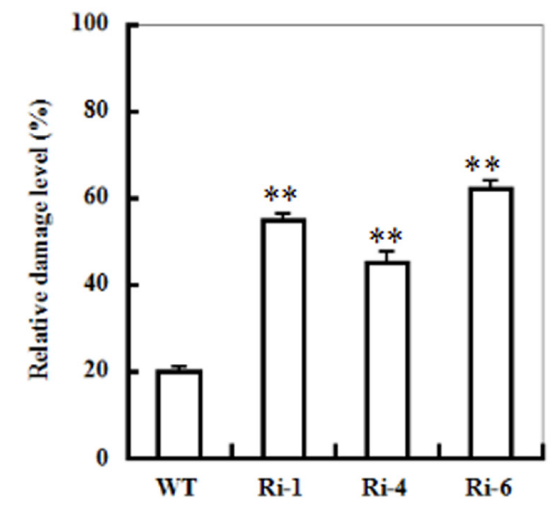

C

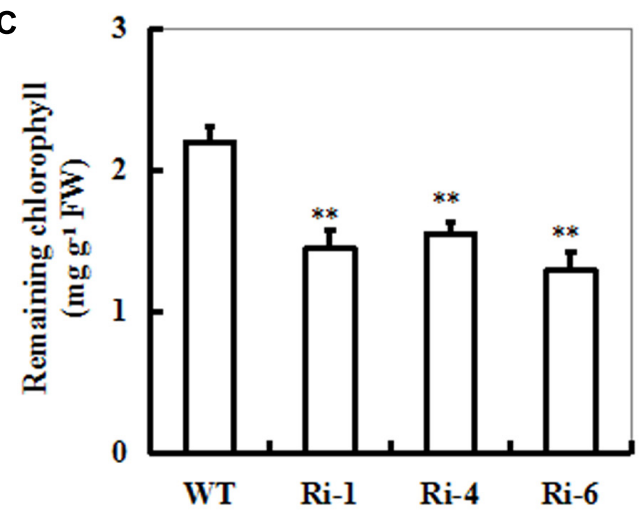

FIGURE 3 | Responses of wild-type and SiR under-expressing plants to oxidative stress. (A) Toxic effect of MV on WT and SiR RNAi plants. Five-Week-old WT and RNAi lines (Ri-1, Ri-4 and Ri-6) were sprayed with $20 \mu \mathrm{M}$ of MV and examined 3 days later. (B) Relative damage level in the wild-type and RNAi plants after MV exposure. Values are mean $\pm \mathrm{SE}, n=20 .{ }^{*} t$-test, with $P<0.01$. (C) Residual chlorophyll in the wild-type and RNAi lines after MV exposure. Values are mean $\pm \mathrm{SE}, n=20$. ${ }^{* *}$ t-test, with $P<0.01$

contents were determined in treated and control leaves from wild-type and $S i R$ under-expressing plants. The effect of MV on total sulfite levels in these $S i R$ under-expressing lines resulted in relatively bigger increases of $61-73 \%(66,61$, and $73 \%$ increases for RNAi-1, -4, and -6, respectively; Figure 5A). By contrast, the sulfite content in the leaves of WT plants only increases by $36 \%$ upon MV exposure (Figure 5A). In addition, under control conditions, sulfite levels in the RNAi lines had no significant increase compared to the wild-type plants (Figure 5A).

For changes in the GSH content, a significant increase was detected in the wild-type plants $(60 \%$ increase), but not in the three RNAi lines (only nearly 30\% increase on average; Figure 5B). Noticeably, under control conditions, total GSH levels in these RNAi lines had significant reductions compared to the wild-type plants (Figure 5B). These results demonstrated that reduced $S i R$ expression resulted in excess sulfite accumulation and insufficient biosynthesis of GSH, indicating that $S i R$ was involved in MV-induced oxidative stress tolerance largely attributable to GSH levels in plants.

\section{SiR Knockdown-Triggered Sensitivity to Oxidative Stress Was Reversed by GSH}

The MV-induced loss of chlorophyll and accelerated cellular damage in SiR-impaired plants may largely result from the insufficient GSH levels (Figures 4 and 5). To examine this directly, 1-week-old seedlings from WT and SiR mutants (Ri-4 and Ri-6) were treated with MV or MV plus GSH for a week. MV exposure caused marked reductions in chlorophyll and survival rates that were alleviated in the presence of GSH in both wild-type and RNAi lines (Figure 6A). Interestingly, the WT plants exhibited the lowest chlorophyll degradation and the highest survival of seedlings (Figures 6B,C). This observation indicates that GSH may play a protective role in MV-induced oxidative stress. 


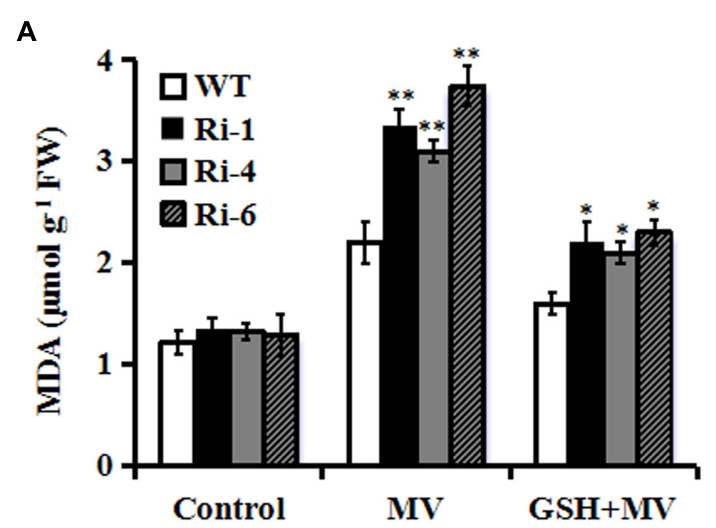

B

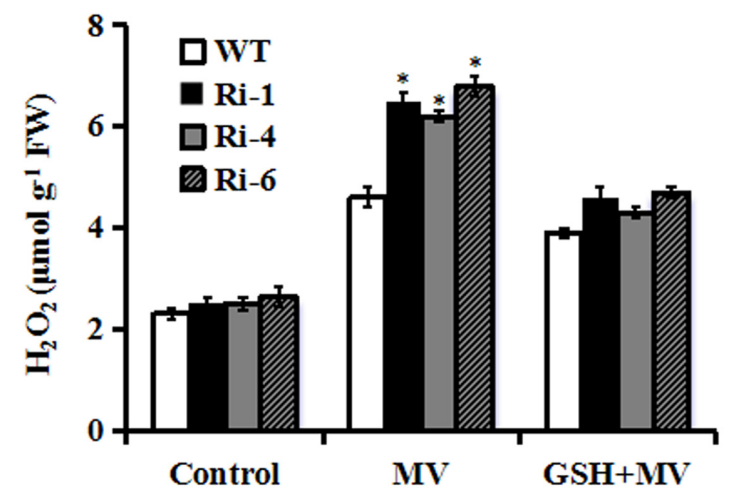

FIGURE 4 | Changes of MDA and $\mathrm{H}_{2} \mathrm{O}_{2}$ in SiR RNAi lines under oxidative stress. (A) Determination of MDA accumulation in leaves of WT and three RNAi lines 3 days after MV $(20 \mu \mathrm{M})$ or GSH $(10 \mathrm{mg} / \mathrm{L})$ plus $\mathrm{MV}(2 \mathrm{O} \mu \mathrm{M})$ spraying. (B) Quantitative determination of $\mathrm{H}_{2} \mathrm{O}_{2}$ accumulation in leaves of WT and three RNAi lines 3 days after MV $(20 \mu \mathrm{M})$ or GSH (10 mg/L) plus MV $(20 \mu \mathrm{M})$ spraying. In both (A,B), each experiment was repeated twice. Bar indicates SE. Values are mean $\pm \mathrm{SE}$. ${ }^{*} t$-test, with $P<0.01$; ${ }^{*} t$-test, with $P<0.05$.

\section{Changes in Sulfite Metabolism-Related Gene Expression in WT and SiR Under-Expressing Lines upon MV Exposure}

The transcripts of sulfite metabolism-related enzymes sulfite oxidase (SO), UDP- sulfoquinovose synthase (SQS1), and adenosine-5'-phosphosulfate reductase (APR1, APR2 and APR3) were monitored upon MV exposure in the wild-type and $S i R$ under-expression plants by qRT-PCR. After MV exposure for $12 \mathrm{~h}$, the transcripts of all the genes except for the SQS1 displayed similarly increasing trends between the WT and RNAi plants (Figure 7). In particular, enhanced expression of the APR transcripts was quite evident in SiRimpaired plants compared with those in wild-type plants. Moreover, the transcripts of the APR1 were significantly up-regulated even under normal conditions (Figures 7D-F). This suggests that the increased sulfite in the SiR-impaired plants could be a result of the reduced $\mathrm{SiR}$ coupled to

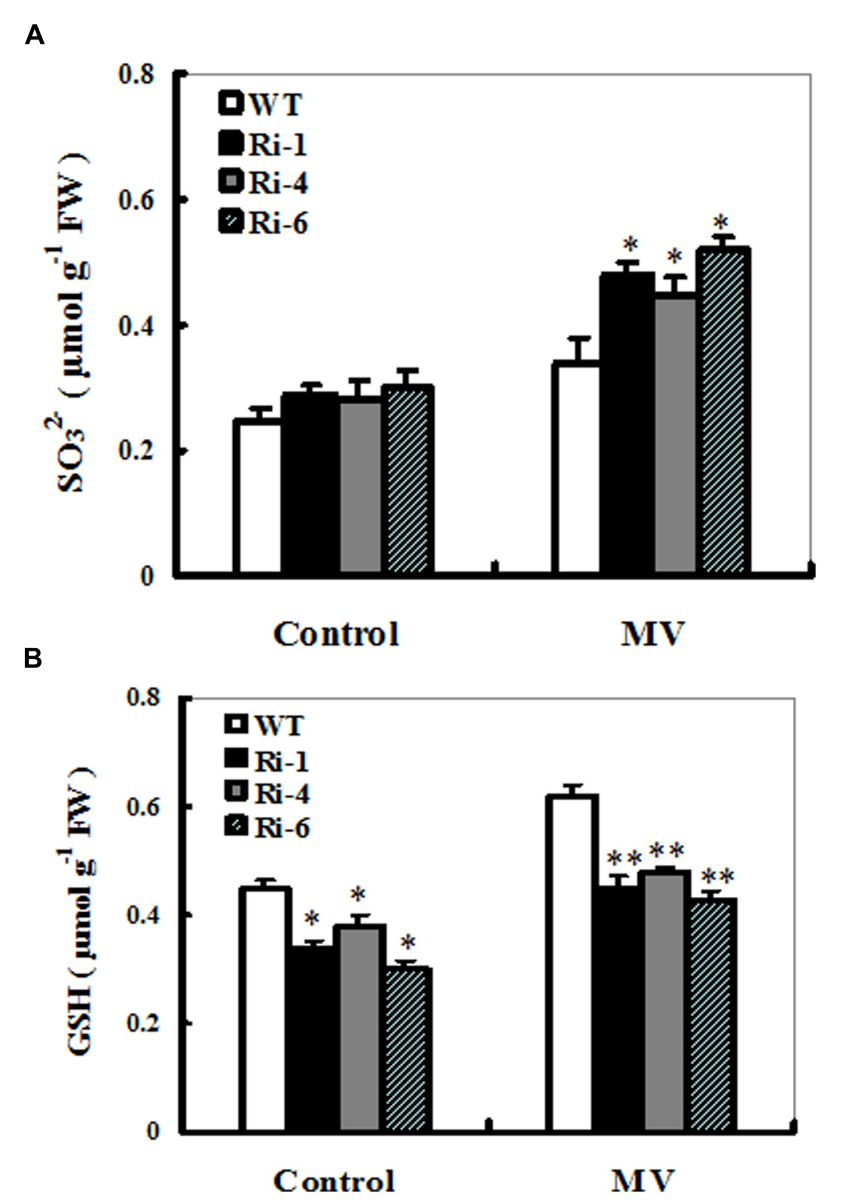

FIGURE 5 | Sulfite and glutathione accumulations in the wild-type and SiR RNAi plants upon MV exposure. Contents of sulfite (A) and glutathione (B) were measured 3 days after MV $(20 \mu \mathrm{M})$ spraying. Each experiment was repeated three times. Bar indicates SE. Values are mean $\pm \mathrm{SE}$. $* * t$-test, with $P<0.01$; t-test, with $P<0.05$.

enhanced APR expression during control and oxidative stress.

\section{DISCUSSION}

As a key enzyme in the sulfate assimilation pathway, $\mathrm{SiR}$ is not only involved in plant growth and development, but in stress response (Khan et al., 2010; Yarmolinsky et al., 2013, 2014). Our genetic evidence suggests that SiR participates in oxidative stress tolerance by possibly regulating sulfite homeostasis, GSH levels and sulfite metabolism-related gene expression.

\section{SiR is Evolutionarily Conserved and Functionally Divergent in Plant Species}

For the SiR, there exists one copy in Arabidopsis genome and two copies in the genomes of rice and poplar (Bork et al., 1998; Kopriva, 2006). A phylogenetic tree was established 
A

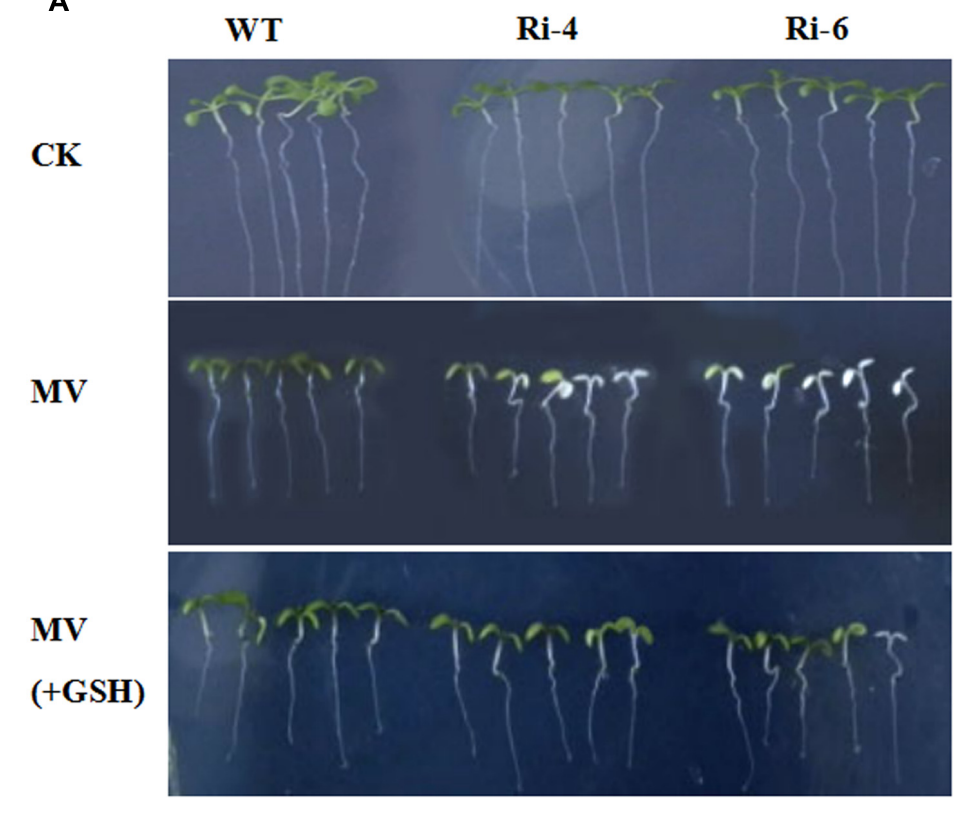

B

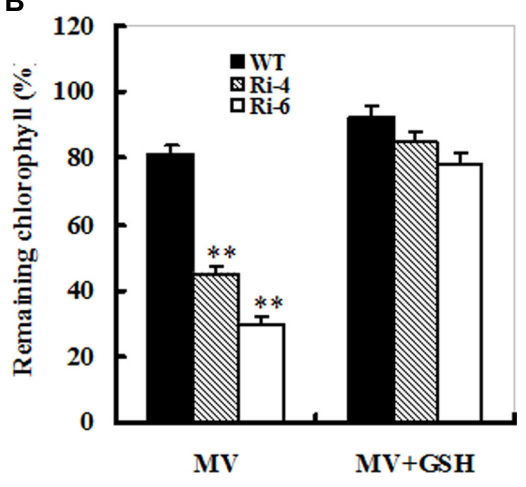

C

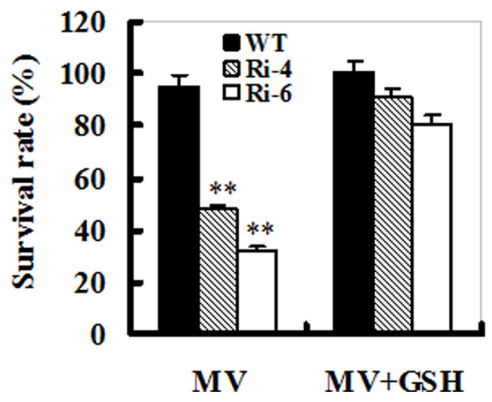

FIGURE 6 | Effect of exogenous GSH on response of wild-type and SiR under-expressing plants to oxidative stress. (A) Representative growth phenotypes of WT and RNAi seedlings when exposed to MV and MV+GSH. Seven-day-old seedlings of WT and RNAi lines (Ri-4 and -6) were vertically growing on 1/2 MS medium supplemented with 0 (CK), MV(5 $\mu \mathrm{M})$, and MV $(5 \mu \mathrm{M})$ plus GSH (10 mg/L) for 7 days. (B) Relative residual chlorophyll (\%) in the wild-type and RNAi lines after MV and MV plus GSH treatments. Values are mean $\pm \mathrm{SE}, n=15$. ${ }^{* *} t$-test, with $P<0.01$. (C) Survival rates (\%) under MV stress in (B) were determined as the number of visibly green plants after 7 days. Values are mean $\pm \mathrm{SE}, n=30$. $* *$-test, with $P<0.01$.

based on $\mathrm{SiR}$ protein sequences available in GenBank from 10 plant species including Arabidopsis, tobacco, tomato, soybean, castor, rice, barley, wild wheat, sorghum, and maize (Supplementary Figure S1). This indicates that SiR is evolutionarily conserved in plant species. $\mathrm{SiR}$ was found to be expressed in nearly all tissue types in Arabidopsis (Figure 1A), indicating this gene may be constitutively expressed during both vegetative and reproductive growth. Besides leaves, higher transcript levels of the $S i R$ were observed in siliques; suggesting that SiR-dependent sulfate assimilation may be involved in sulfur-containing protein biosynthesis during seed development in Arabidopsis. Interestingly, relative higher levels of $S i R$ transcripts were also found in roots, indicating that $S i R$ may play a role in response to nutritional stress such as sulfate deficiency in roots. This notion was supported by the clue that SiR mRNA levels were up-regulated when Arabidopsis plants were subjected to sulfate starvation (Hell, 1997). Several lines of evidence have recently shown that plant $\mathrm{SiR}$ is essential for growth and development (Khan et al., 2010) and participates in sulfite stress response and leaf senescence (Yarmolinsky et al., 2013, 2014); Also, SiR was confirmed to be involved in oxidative stress response in Arabidopsis (This study). Taken these findings together, it is strongly evidenced that plant $\mathrm{SiR}$ is functionally divergent.

\section{SiR-Dependent Sulfite Reduction is Indispensable for Sulfite Homeostasis during Oxidative Stress}

SO, SiR, SQS1, and APR are key enzymes that catalyze the diversion of sulfite to other assimilatory pathways (Eilers et al., 2001; Benning, 2007; Brychkova et al., 2013). In this study, transcript levels of the three APR genes (APR1, APR2, and $A P R 3)$ were significantly up-regulated in the $S i R$-impaired lines during $\mathrm{MV}$-induced oxidative stress compared to those in the WT plants (Figures 7D-F). Moreover, sulfite content significantly increased in the leaves of the SiR knock-down lines (Figure 5A). These results suggest that toxic sulfite levels were increasingly accumulated in plant cells during oxidative stress because of enhanced $A P R$ expression and impaired SiR. This observation could be supported by the evidence that $\mathrm{SiR}$ mutant RNAi plants were severely suffered by $\mathrm{SO}_{2}$ stress due to excess sulfite accumulation (Yarmolinsky et al., 2013). Besides SiR-dependent sulfite reduction pathway, sulfite can be incorporated into sulfolipids, which is catalyzed by the chloroplast-localized UDP-sulfoquinovose synthase (SQS1). Noticeably, transcript levels of the SQS1 were not changed significantly in both WT and SiR under-expressing plants upon MV exposure (Figure 7C), indicating that sulfolipid synthesis in plastids may not be a predominant pathway for sulfite 

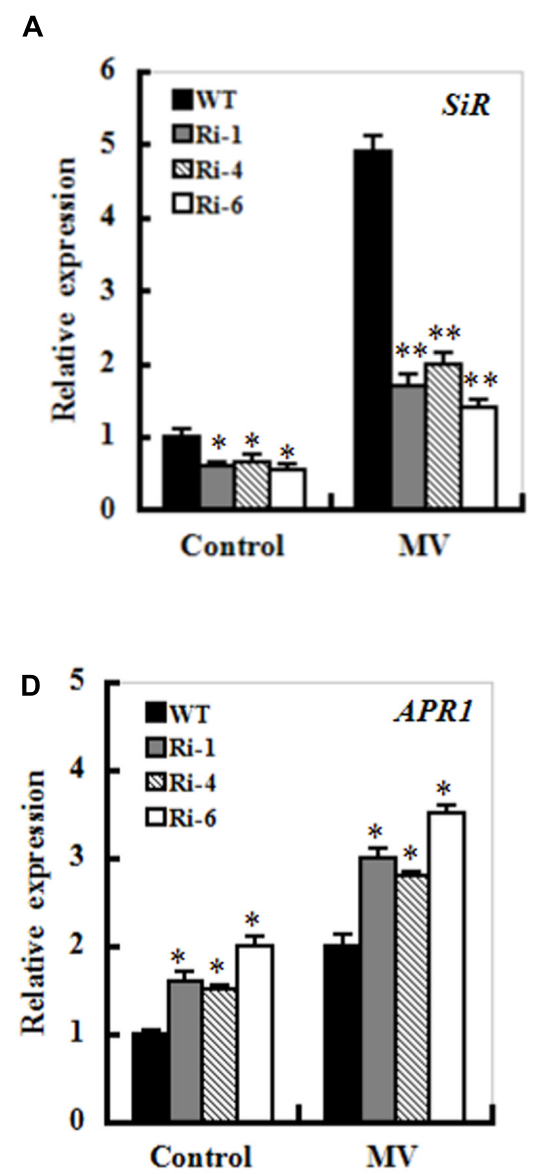
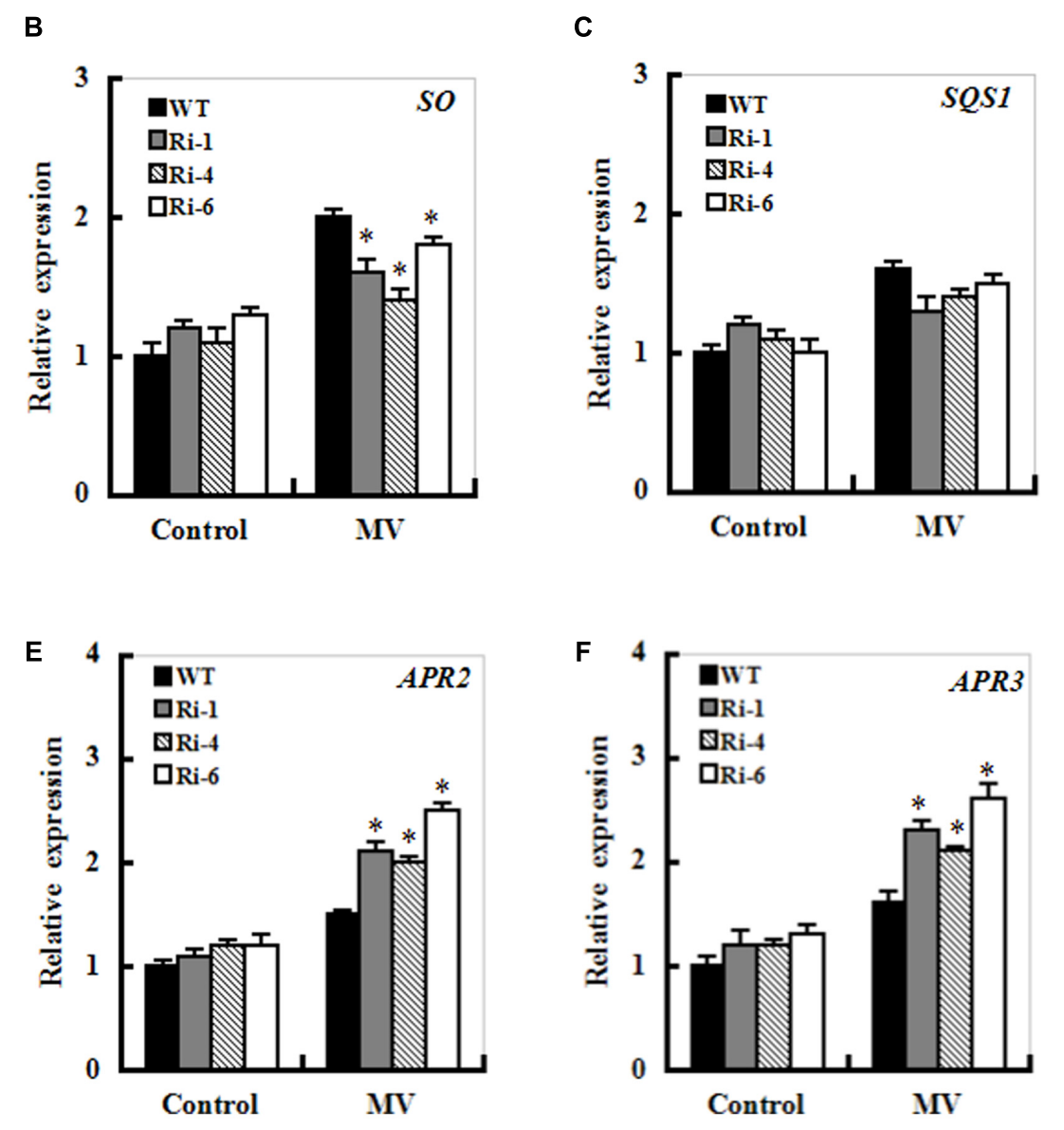

FIGURE 7 | Effect of SiR suppression on transcripts of the sulfite network genes under oxidative stress. Leaf samples from 5-week-old WT and three RNAi lines were harvested after 12 h of MV (20 $\mu \mathrm{M})$ spraying and transcriptional expression of SiR (A), SO (B), SQD1 (C), APR1 (D), APR2 (E), and APR3 (F) was detected by qRT-PCR. mRNA levels of these genes were normalized to the transcripts of Actin2 in the same samples. For each assay, the expression level of WT under control conditions was taken as 1.0, and data represented mean \pm SE of three biological replicates. ${ }^{* *} t$-test, with $P<0.01$; ${ }^{*} t$-test, with $P<0.05$.

homeostasis during oxidative stress. Although transcript levels of $S O$ were up-regulated upon MV exposure, SiR-impaired plants showed more severe damage than the WT. These results demonstrate that $\mathrm{SiR}$ is indispensable for maintaining sulfite homeostasis during oxidative stress. Further work is needed to dissect the SiR-dependent and SO-dependent sulfite homeostasis networks in SiR and SO mutants using transcriptome, proteome, or metabolome approaches.

\section{Glutathione Plays an Important Role in Response to Oxidative Stress for SiR-Impaired Plants}

Methyl viologen (MV, paraquat) is one of the most widely used herbicides in agriculture. It rapidly enters leaves and then chloroplasts, where it disrupts PSI electron transport, reducing oxygen to ROS. Thus, it can lead to cell toxicity, membrane peroxidation and even cell death. Sulfite is reduced by $\mathrm{SiR}$ to form sulfide, which is then incorporated into Cys and other sulfur-containing compounds such as GSH. As a major organic thiol-containing metabolite, GSH has an important role in maintaining redox homeostasis (Nagalakshmi and Prasa, 2001).

In this study, higher hydrogen peroxide and MDA levels, which are hallmarks of oxidative stress were detected upon $\mathrm{MV}$ exposure in the $\mathrm{SiR}$ knock-down transgenic lines (Figure 4). Further investigations showed that a significant increase in the GSH content was detected in the WT, but not in the SiR under-expressing lines (Figure 5B). This indicates that amounts of GSH were influenced by the SiR levels and in return, GSH levels affected oxidative stress response of the $S i R$-impaired plants directly. In addition, the accumulation of hydrogen peroxide in these RNAi lines may also be a consequence of the lower GSH levels, which weakened the antioxidant capacity. In support of this viewpoint, Ding et al. (2009) showed that impairment of glutathione reductase (GR) led to enhanced sensitivity to $\mathrm{MV}$-induced oxidative stress in transgenic tobacco because of the reduced capacity for regeneration of GSH (Ding et al., 2009). 
In summary, $\mathrm{SiR}$ can protect plants from oxidative stress, and has a potential for application to economically important crop plants. Future work will be needed to dissect the mechanisms in detail by which the SiR-regulated sulfate reductive pathway involved in $\mathrm{MV}$-induced oxidative stress response using mutants of sulfite metabolism-related genes in Arabidopsis.

\section{AUTHOR CONTRIBUTIONS}

$\mathrm{ZX}$ designed the research. MW, YJ, ZXu, and ZX performed research and conducted data analyses. ZX wrote the manuscript.

\section{ACKNOWLEDGMENTS}

This work was financially supported by the National Natural Science Foundation of China (30971548) and Project of

\section{REFERENCES}

Alscher, R. G. (1989). Biosynthesis and antioxidant function of glutathione in plants. Physiol. Plant. 77, 457-464. doi: 10.1111/j.1399-3054.1989.tb05667.x

Benning, C. (2007). Questions remaining in sulfolipid biosynthesis: a historical perspective. Photosynth. Res. 92, 199-203. doi: 10.1007/s11120-007-9144-6

Bork, C., Schwenn, J. D., and Hell, R. (1998). Isolation and characterization of a gene for assimilatory sulfite reductase from Arabidopsis thaliana. Gene 212, 147-153. doi: 10.1016/S0378-1119(98)00155-3

Bradford, M. M. (1976). A rapid and sensitive method for the quantification of microgram quantities of protein utilizing the principal of protein-dye binding. Anal. Biochem. 72, 248-254. doi: 10.1016/0003-2697(76)90527-3

Brychkova, G., Grishkevich, V., Fluhr, R., and Sagi, M. (2013). An essential role for tomato sulfite oxidase and enzymes of the sulfite network in maintaining leaf sulfite homeostasis. Plant Physiol. 161, 148-164. doi: 10.1104/pp.112.208660

Brychkova, G., Xia, Z., Yang, G., Yesbergenova, Z., Zhang, Z., Davydov, O., et al. (2007). Sulfite oxidase protects plants against sulfur dioxide toxicity. Plant J. 50, 696-709. doi: 10.1111/j.1365-313X.2007.03080.x

Brychkova, G., Yarmolinsky, D., Fluhr, R., and Sagi, M. (2012). The determination of sulfite levels and its oxidation in plant leaves. Plant Sci. 190, 123-130. doi: 10.1016/j.plantsci.2012.04.004

Clough, S. J., and Bent, A. F. (1998). Floral dip: a simplified method for Agrobaterium -mediated transformation of Arabidopsis thaliana. Plant J. 16, 735-743. doi: 10.1046/j.1365-313x.1998.00343.x

Ding, S., Lu, Q., Zhang, Y., Yang, Z., Wen, X., Zhang, L., et al. (2009). Enhanced sensitivity to oxidative stress in transgenic tobacco plants with decreased glutathione reductase activity leads to a decrease in ascorbate pool and ascorbate redox state. Plant Mol. Biol. 69, 577-592. doi: 10.1007/s11103-0089440-3

Draper, H. H., and Hadley, M. (1990). Malondialdehyde determination as index of lipid peroxidation. Meth. Enzymol. 86, 421-431. doi: 10.1016/0076-6879(90) $86135-\mathrm{I}$

Eilers, T., Schwarz, G., Brinkmann, H., Witt, C., Richter, T., Nieder, J., et al. (2001). Identification and biochemical characterization of Arabidopsis thaliana sulfite oxidase. A new player in plant sulfur metabolism. J. Biol. Chem. 276, 46989-46994. doi: 10.1074/jbc.M108078200

Gechev, T. S., Van Breusegem, F., Stone, J. M., Denev, I., and Laloi, C. (2006). Reactive oxygen species as signals that modulate plant stress responses and programmed cell death. Bioessays 28, 1091-1101. doi: 10.1002/bies.20493

Griffith, O. W. (1980). Determination of glutathione and glutathione disulfide using glutathione reductase and 2-vinylpyridine. Anal. Biochem. 106, 207-212. doi: 10.1016/0003-2697(80)90139-6

Hell, R. (1997). Molecular physiology of plant sulfur metabolism. Planta 202, 138-148. doi: $10.1007 /$ s004250050112
Innovative Talents of Science and Technology of He'nan Educational Committee (16HASTIT021).

\section{SUPPLEMENTARY MATERIAL}

The Supplementary Material for this article can be found online at: http://journal.frontiersin.org/article/10.3389/fpls.2016.01843/ full\#supplementary-material

\section{FIGURE S1 | Phylogenetic tree based on the amino acid sequence} alignment of plant SiRs. These plant species include Arabidopsis thaliana (CAA89154.1), Solanum lycopersicum (AFB83709.1), Ricinus communis (XP_002513495.1), Nicotiana benthamiana (ACN23794.1), Glycine max (XP_003540209.1), Sorghum bicolor (XP_002441346.1), Oryza sativa (NP_001055978.1), Hordeum vulgare (BAK03240.1), Brachypodium distachyon (XP_003568157.1) and Zea Mays (NP_001105302.1). The bootstrap values shown were calculated based on 500 replications. The tree was constructed using the neighbor-joining method.

Huo, Y., Wang, M., Wei, Y., and Xia, Z. (2016). Overexpression of the maize psbA gene enhances drought tolerance through regulating antioxidant system, photosynthetic capability, and stress defense gene expression in tobacco. Front. Plant Sci. 6:1223. doi: 10.3389/fpls.2015.01223

Kang, Y. W., Lee, J. Y., Jeon, Y., Cheong, G. W., Kim, M., and Pai, H. S. (2010). In vivo effects of $\mathrm{NbSiR}$ silencing on chloroplast development in Nicotiana benthamiana. Plant Mol. Biol. 72, 569-583. doi: 10.1007/s11103-009-9593-8

Khan, M. S., Haas, F. H., Samami, A. A., Gholami, M., Bauer, A., Fellenberg, K., et al. (2010). Sulfite reductase defines a newly discovered bottleneck for assimilatory sulfate reduction and is essential for growth and development in Arabidopsis thaliana. Plant Cell 22, 1216-1231. doi: 10.1105/tpc.110.074088

Kopriva, S. (2006). Regulation of sulfate assimilation in Arabidopsis and beyond. Ann. Bot. 97, 479-495. doi: 10.1093/aob/mcl006

Lang, C., Popko, J., Wirtz, M., Hell, R., Herschbach, C., Kreuzwieser, J., et al. (2007). Sulphite oxidase as key enzyme for protecting plants against sulphur dioxide. Plant Cell Environ. 30, 447-455. doi: 10.1111/j.1365-3040.2006. 01632.x

Leustek, T., Martin, M. N., Bick, J. A., and Davies, J. P. (2000). Pathways and regulation of sulfur metabolism revealed through molecular and genetic studies. Annu. Rev. Plant Physiol. Plant Mol. Biol. 51, 141-165. doi: 10.1146/annurev. arplant.51.1.141

Leustek, T., and Saito, K. (1999). Sulfate transport and assimilation in plants. Plant Physiol. 120, 637-644. doi: 10.1104/pp.120.3.637

Lewandowska, M., and Sirko, A. (2008). Recent advances in understanding plant response to sulfur-deficiency stress. Acta Biochem. Pol. 55, 457-471.

Livak, K. J., and Schmittgen, T. D. (2001). Analysis of relative gene expression data using real-time quantitative PCR and the $2^{-\Delta \Delta C_{\mathrm{T}}}$ method. Methods 25, 402-408. doi: 10.1006/meth.2001.1262

Nagalakshmi, N., and Prasa, M. N. (2001). Responses of glutathione cycle enzymes and glutathione metabolism to cooper stress in Scenedesum bijugatns. Plant Sci. 160, 291-299. doi: 10.1016/S0168-9452(00)00392-7

Nakayama, M., Akashi, T., and Hase, T. (2000). Plant sulfite reductase: molecular structure, catalytic function and interaction with ferredoxin. J. Inorg. Biochem. 82, 27-32. doi: 10.1016/S0162-0134(00)00138-0

Noctor, G., Arisi, A. M., Jouanin, L., Kunert, K. J., Rennenberg, H., and Foyer, C. H. (1998). Glutathione: biosynthesis, metabolism and relationship to stress tolerance explored in transformed plants. J. Exp. Bot. 49, 623-647. doi: 10.1093/ jexbot $/ 49.321 .623$

Sekine, K., Fujiwara, M., and Nakayama, M. (2007). DNA binding and partial nucleoid localization of the chloroplast stromal enzyme ferredoxin: sulfite reductase. FEBS J. 274, 2054-2069. doi: 10.1111/j.1742-4658.2007.05748.x

Wirtz, M., Droux, M., and Hell, R. (2004). O-acetylserine (thiol) lyase: an enigmatic enzyme of plant cysteine biosynthesis revisited in Arabidopsis thaliana. J. Exp. Bot. 5, 785-1798. 
Xia, Z., Huo, Y., Wei, Y., Chen, Q., Xu, Z., and Zhang, W. (2016). The Arabidopsis LYST INTERACTING PROTEIN 5 acts in regulating abscisic acid signaling and drought response. Front. Plant Sci. 7:758. doi: 10.3389/fpls.2016. 00758

Xia, Z., Sun, K., Wang, M., Wu, K., and Zhang, H. (2012). Overexpression of a maize sulfite oxidase gene in tobacco enhances tolerance to sulfite stress via sulfite oxidation and CAT-mediated $\mathrm{H} 2 \mathrm{O} 2$ scavenging. PLoS ONE 7:e37383. doi: 10.1371/journal.pone.0037383

Yarmolinsky, D., Brychkova, G., Fluhr, R., and Sagi, M. (2013). Sulfite reductase protects plants against sulfite toxicity. Plant Physiol. 161, 725-743. doi: 10.1104/ pp.112.207712

Yarmolinsky, D., Brychkova, G., Kurmanbayeva, A., Bekturova, A., Ventura, Y., Khozin-Goldberg, I., et al. (2014). Impairment in sulfite reductase leads to early leaf senescence in tomato plants. Plant Physiol. 165, 1505-1520. doi: 10.1104/ pp.114.241356

Conflict of Interest Statement: The authors declare that the research was conducted in the absence of any commercial or financial relationships that could be construed as a potential conflict of interest.

Copyright (๑) 2016 Wang, Jia, Xu and Xia. This is an open-access article distributed under the terms of the Creative Commons Attribution License (CC BY). The use, distribution or reproduction in other forums is permitted, provided the original author(s) or licensor are credited and that the original publication in this journal is cited, in accordance with accepted academic practice. No use, distribution or reproduction is permitted which does not comply with these terms. 